\title{
Strategy for the Control of a Dual-stage Nano-positioning System with a Single Metrology*
}

\author{
Yvan Michellod, Philippe Mullhaupt and Denis Gillet \\ Laboratoire d'Automatique, École Polytechnique Fédérale de Lausanne (EPFL), \\ CH-1015 Lausanne, Switzerland. \\ Email: yvan.michellod@epfl.ch
}

\begin{abstract}
A double-stage feedback control structure for a double-stage mechanical system, with a single optical metrology is developed to reach nanometer accuracy at high bandwidth over large displacements. A piezoelectric stack actuator is used for fine positioning, while a permanent magnet (PM) stepper motor handles the coarse positioning.

Two different control approaches are compared for driving the PM stepper motor, while a classical PID controller is designed to drive the piezoelectric actuator. Since only a single measurement device is used, the references for both control loops (fine and coarse) must be appropriately obtained. An adequate control structure including a partial observer is designed so as to take into account the influence of the fine actuator on the position estimation of the coarse actuator. The complete control mechanism and strategy ensure the tracking of the real reference with sufficient accuracy and bandwidth.
\end{abstract}

Keywords-Dual-stage, Dual-input Single-output, Nanopositioning, Large-displacement, Trajectory tracking, Observer, Feedback, Piezoelectric, PM stepper motor.

\section{INTRODUCTION}

Large-displacement nanometer-accuracy position-tracking systems are becoming more and more attractive with increasinng miniaturization (see [1], [2], [3], [4], [5], [6] for examples).

However, the translation range and/or bandwidth of such systems is often limited by the tradeoff between actuator imperfections and difficulties in finding suitable sensors of reasonable cost so as to close the loop.

Most applications implementing high-resolution fastpositioning systems ([1], [5], [6], [7], [8]) use piezoelectric stack actuators. Indeed, the intrinsic piezoelectric characteristics make such actuators most suitable for fast and fine positioning over a very short stroke. Since this short stroke is not sufficient for the application considered in this paper, it is proposed to combine the piezoelectric actuator with a secondary stage consisting of a PM stepper motor. Classical control approaches for a PM stepper motor (which shows highly nonlinear dynamics) use the Park transformation to modify the considered referential ([10], [11], [12], [13], [14]). $H_{\infty}$ control with a simplified model ([10]), state feedback linearization ([11], [12]), or passivity and flatness-based controllers ([16]) are the main modern control methodologies

*This work has been supported partly by the Swiss National Science Foundation. proposed. Stepper motors are also often controlled statically through high-level interfaces for precise positioning.

The use of a double-stage structure is proposed to help overcoming the intrinsic drawbacks of single actuators. A similar approach, on a smaller scale, combining the effect of a fine piezoelectric with a coarse voice-coil has been recently proposed for Hard Disk Drives ([17], [18], [19], [20], [21]).

The global control structure proposed below combines optimally the effect of the two stages on the controlled output position to reach nanometer-accuracy trajectory tracking over several centimeters.

The paper is organized as follows. Section II introduces the control problem. Then, two different permanent magnet stepper motor controllers are designed in Section III. In Section $\mathrm{IV}$, the fine stage controller is designed, and the continuous global controller which guarantees coordinated action of both actuators, together with achieving the required specifications, is described in Section V. Finally, some experimental results are presented.

\section{CONTROL PROBLEM}

In order to improve the resolution of the Very Large Telescope Interferometer (VLTI) at the European Southern Observatory (ESO), by rejecting the atmospheric perturbation, a mechanical system realizing a differential delay line is under study. The atmospheric influence is considered as a stochastic perturbation. It is measured and processed indirectly by a fringe sensor unit (which is not detailed herein) so as to provide the reference trajectory of our positioning system.

To achieve nanometer accuracy over both a high-frequency bandwidth and a large total spatial displacement (several centimeters), a double-stage system combining a piezoelectric stack actuator and a permanent magnet (PM) stepper motor is proposed. The piezoelectric (PZT) stack actuator is indeed the only actuator with the required bandwidth and accuracy. However, its main drawback is its limited stroke. Therefore, it is proposed to combine mechanically its action on the output optics position with a coarse permanent magnet (PM) stepper motor. Although the stepper motor presents a lower accuracy and bandwidth, it is not stroke-limited. Hence, each actuator compensates for the weak point of the other one. The difficulty lies in coordinating the control action of both actuators so as to maximize their respective advantages. This will be detailed 


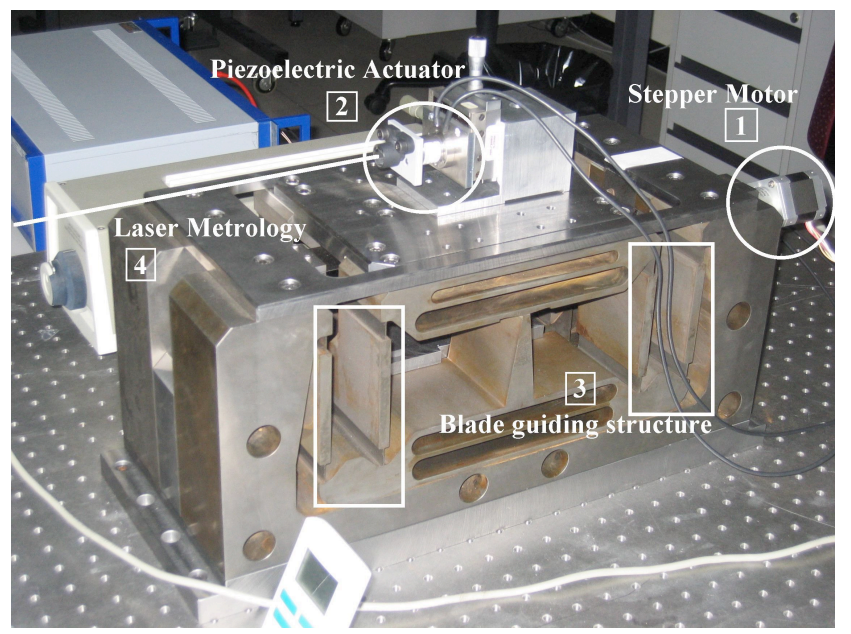

Fig. 1. Experimental setup: 1. Stepper motor, driving the blade stage; 2 Piezoelectric actuator, driving the fine stage; 3 . Double parallelogram flexure with notch-hinges ensuring a straight and flat movement over the whole stroke; 4. Laser metrology.

in Section V. The mechanical concept (a double parallelogram flexure with notch hinges) is shown in Figure 1, where both actuators are controlling the single translation axis $z$. The two actuator outputs are summed (i.e. mechanically mounted in series, but controlled in parallel) to form the overall controlled output, which is supposed to track a stochastic reference with a frequency content up to $200 \mathrm{~Hz}$.

A single interferometer reads the output optics position resulting from the combination of the two stages. Hence, there are more actuators than the number of controlled output positions. The proposed control strategy coordinates simultaneousely both actuators without resorting to a hybrid switching control strategy (where actuators are switched on and off so as to achieve the desired accuracy).

\section{A. Actuator choice}

Since the piezoelectric (PZT) stack actuator is indeed the only actuator with the requested bandwidth and accuracy, the piezoelectric S-325 from "Physic Instrumente" (PI) with a stroke of $30 \mu \mathrm{m}$ and a bandwidth of approximatively $300 \mathrm{~Hz}$ was selected (in fact, the bandwidth is limited by the optics used to sense the actual position, not by the piezoelectric itself). Its main drawback (apart from its hysteresis which can be overcome by an appropriate controller) is its relative short stroke. In fact, the piezoelectric effect implies a tradeoff between the bandwidth and the obtainable length. Therefore, the main characteristic of the coarse actuator should be a long stroke to compensate the fine actuator limitations: a PM stepper motor NEMA 17 from "Ultramotion" combined with a leadscrew presenting a full course of $70 \mathrm{~mm}$ was selected.

\section{PM STEPPER MOTOR CONTROLLER DESIGN}

\section{A. Microstepping driver}

The classical approach to drive a PM stepper motor at high resolution is to use a microstepping driver, which deals with the motor coils. This microstepping device divides the mechanical steps of the motor itself by injecting current in several coils simultaneously: the rotor simply aligns itself with the magnetic field generated by the stator coils.

This high-resolution electronic driver has the advantage of being easily controlled with digital pulses, since each pulse corresponds to a single microstep (of about $40 \mathrm{~nm}$ with the 256 microstep driver IM483H and the NEMA 17 stepper motor selected).

So as to track unpredictable stochastic trajectories, it is proposed to close the loop on the estimated position error of the coarse stage (see Section V). Since the coarse actuator perturbs the overall controlled output with its actions, a hybridlike nonlinear control law is proposed to minimize its action. The coarse controller input signal is evaluated and compared to an absolute threshold and action is taken only when the input signal is greater than a predefined level (see Figure 2). As illustrated in Figure 2, the stepper closed-loop stage reacts

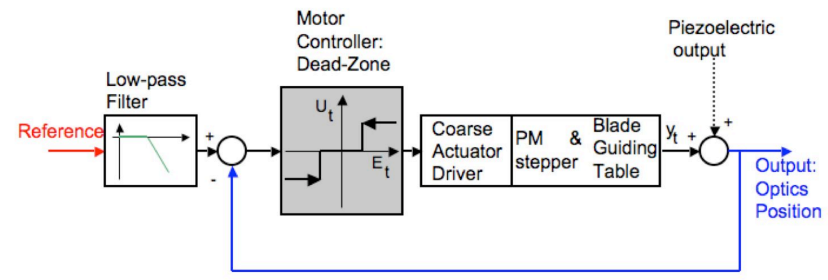

Fig. 2. Structure of the coarse stepper motor controller using the standard electronic driver.

to the error signal only when it is above a certain limit. Thanks to the dead-zone controller, the PZT stack actuator is driven slowly back to its mid-position when getting too close to its stroke limits. Thus, the controller design amounts to fixing the limits and generating the trigger of the coarse moves whenever the coarse stage estimated error is larger than the predefined limits.

The main advantage of such a controller is the ease of implementation of the control algorithm. The controller deals only with digital lines, that are really robust to electromechanical perturbations and it does not require a complicated control algorithm.

\section{B. Direct coil-voltage control}

A more elaborated control approach using the first-principle equations of the PM stepper motor (see [9]), including a sine/cosine voltage control of the coils of the motor is described below.

The PM stepper motor is described by two electrical equations and one mechanical equation ([9], [15]), (see Equations (1) to (4)). The proposed control law is based on the stator generated magnetic field position $x$, see Equations (6) to (9). This angle $\mathrm{x}$ corresponds to the absolute angle between a zero reference of the metrology and the current magnetic field 
position (see Figure 3).

$$
\begin{aligned}
& \text { Coarse stage system equations } \\
\frac{\mathrm{d} \theta}{\mathrm{d} t}= & \omega \\
\frac{\mathrm{d} \omega}{\mathrm{d} t}= & -\frac{K_{m}}{J} i_{a} \sin (p \theta)-\frac{K_{m}}{J} i_{b} \sin (p(\theta-\lambda)) \\
& -\frac{F_{f}}{J} \omega-\frac{T_{L}}{J} \\
\frac{\mathrm{d} i_{a}}{\mathrm{~d} t}= & -\frac{R i_{a}}{L}+\frac{K_{m}}{L} \omega \sin (p \theta)+\frac{u_{a}}{L} \\
\frac{\mathrm{d} i_{b}}{\mathrm{~d} t}= & -\frac{R i_{b}}{L}+\frac{K_{m}}{L} \omega \sin (p(\theta-\lambda))+\frac{u_{b}}{L} \\
y_{T}= & \gamma \theta
\end{aligned}
$$

$$
\begin{aligned}
& \text { Control law } \\
u_{a}= & U_{\text {peak }} \sin (x) \\
u_{b}= & U_{\text {peak }} \cos (x) \\
e_{T}= & \operatorname{Ref}_{T}-\gamma \theta \\
x= & \frac{1}{T_{i}} \int_{0}^{t} e_{T}(t) \mathrm{d} t
\end{aligned}
$$

Where respectively $u_{a}, u_{b}$ are the two voltages applied to the phases of the motor and $i_{a}, i_{b}$ the corresponding currents; $R, L, p, \lambda$ and $K_{m}$ are the characteristics of the motor itself, respectively the phase resistor, the phase inductance, the number of steps per revolution of the motor, the angle between the stator poles and the electromagnetic coefficient; $J$ is the inertial reported to the rotation; $F_{f}$ and $T_{L}$ are respectively the viscous friction and load seen from the rotor; $x$ is the controlled phase of the stator magnetic field; $U_{\text {peak }}$ is the peak voltage applied to the coils of the motor; $y_{T}$ is the linear position of the coarse stage (stepper motor with blade guiding structure) and $\theta$ is the corresponding angle of the rotor.

A very simple second-order model of the PM stepper motor can be considered:

$$
T=\frac{y_{t}}{x}=\frac{\gamma \Omega^{2}}{s^{2}+2 \xi \Omega+\Omega^{2}}
$$

Where $\Omega=2 \pi f$ is the electromagnetic resonant frequency between the rotor and the stator magnetic field position, $\xi$ is the damping of this resonant mode and $\gamma$ is the lead-screw rotation to linear ratio. Classical identification procedures provide the three approximative parameters of this simplified model.

As the system is intrinsically stable in the sense that, neglecting the load, the rotor always aligns itself with the magnetic field generated at the stator (see Figure 3), a simple integral controller, summing continuously the position error will automatically drive the system to the correct position (Equation (9)). Therefore, it is proposed to use a simple slow integral effect in the controller, so as not to excite the approximatively identified resonant mode $\omega$ (see Figure 4).

Moreover, to avoid any de-synchronization between the rotor and the generated magnetic field on which the rotor tends

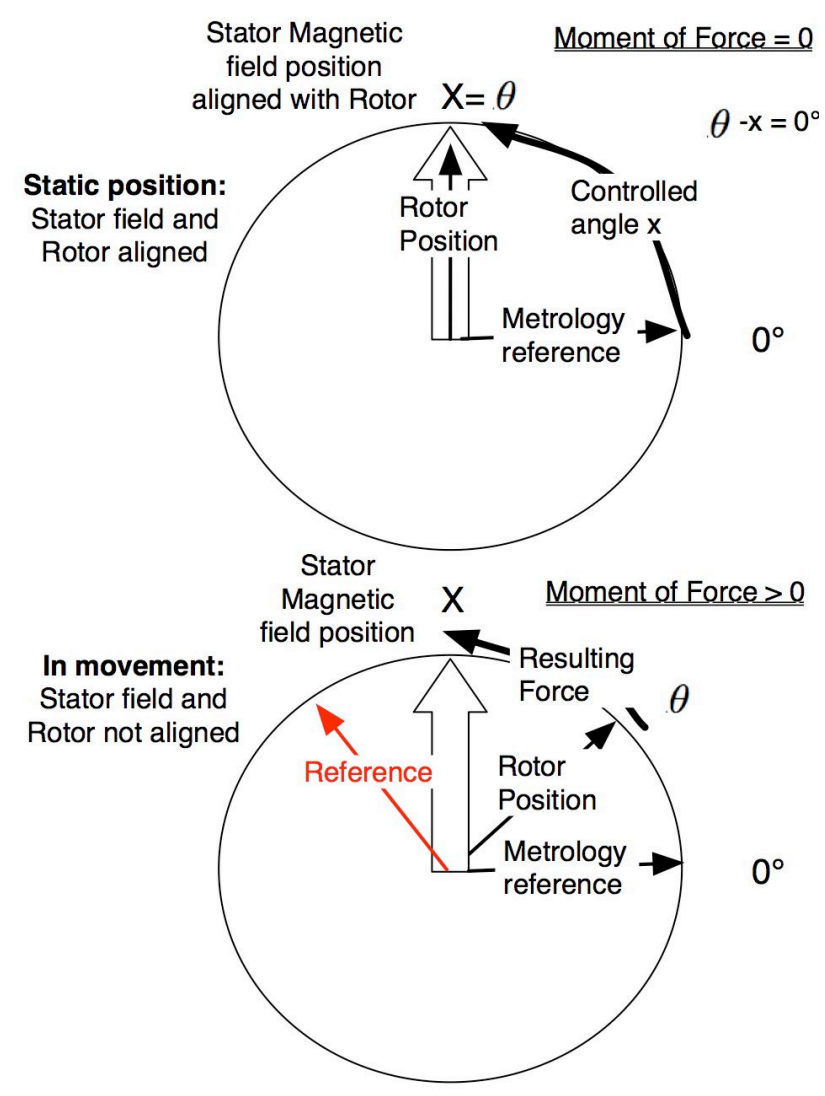

Fig. 3. Direct control of the stepper controlled moment of force. The stator generated magnetic field position induces the moment of rotation applied to the rotor.

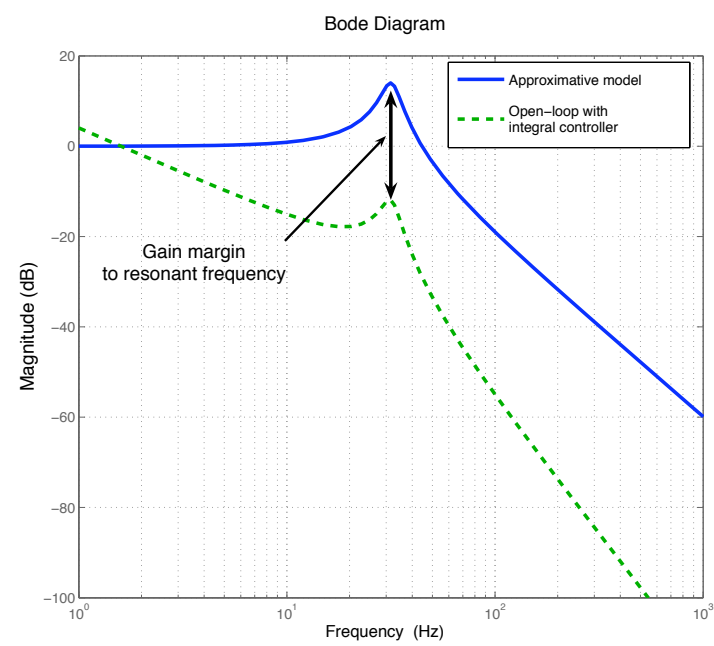

Fig. 4. Simple integral controller for the PM stepper motor trajectory control. The integral gain is adjusted to guarantee a good margin with respect to the electromechanical resonant mode. 
to align, the variation of the controlled angle $x$ is limited.

$$
\dot{x}= \begin{cases}\dot{x}_{\min } & \text { if } \dot{x}<\operatorname{Min} \\ \dot{x} & \text { if Min }<\dot{x}<\operatorname{Max} \\ \dot{x}_{\max } & \text { if } \dot{x}>\operatorname{Max}\end{cases}
$$

This limitation is realized through the saturation of the derivative of $x$, the controlled angular position of the generated magnetic field, so as to ensure that the acceleration is not above the physical limitations of the PM stepper motor.

Furthermore, the coarse stage reference $\operatorname{Ref}_{T}$ (which is fed to the stepper motor controller) is filtered with a third order Butterworth low-pass filter at $10 \mathrm{~Hz}$, so as to both keep only the low frequency content and avoid any excitation of high frequency nonlinear resonant modes (see Figure 5).

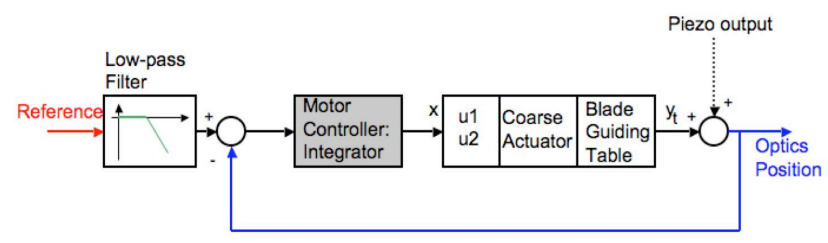

Fig. 5. The coarse PM stepper motor stage.

\section{Comments on the two methods}

The first approach, which relies on a microstepping electronic driver, does not give control on the real trajectory followed by the stepper. However by closing the loop on the final position, the PM stepper motor stage is controlled fairly precisely (depending on the load and on the size of the controller dead-zone). The coarse stage error is compared to its corresponding limits and the controller simply updates the set point of the static position accordingly, making the system approximatively follow the reference of the system.

Since the system tracks unpredictable stochastic trajectories, this behavior is not really adapted. They induce uncertainty on the real movement of the coarse stage. No current model is available to predict the friction, the backlash and dead-zone in transmission with sufficient accuracy to make the trajectory predictable (see Figure 6, curve A). Therefore, this control strategy relies entirely on the capacity of the fine stage actuator to reject the movement of the coarse one.

The second control approach, which relies on a direct coilvoltage control, results in much more predictable movement of the stepper (see Figure 6, curve B), which will be used appropriately to improve the tracking results (see Section IV).

\section{Fine Stage CONTROLleR DESIGN}

The classical identification of the selected piezoelectric stack actuator can be approximated by the following secondorder continuous transfer function (see Figure 7(a)).

$$
P=\frac{y_{p}}{x}=\frac{\omega^{2}}{s^{2}+2 \zeta \omega+\omega^{2}}
$$

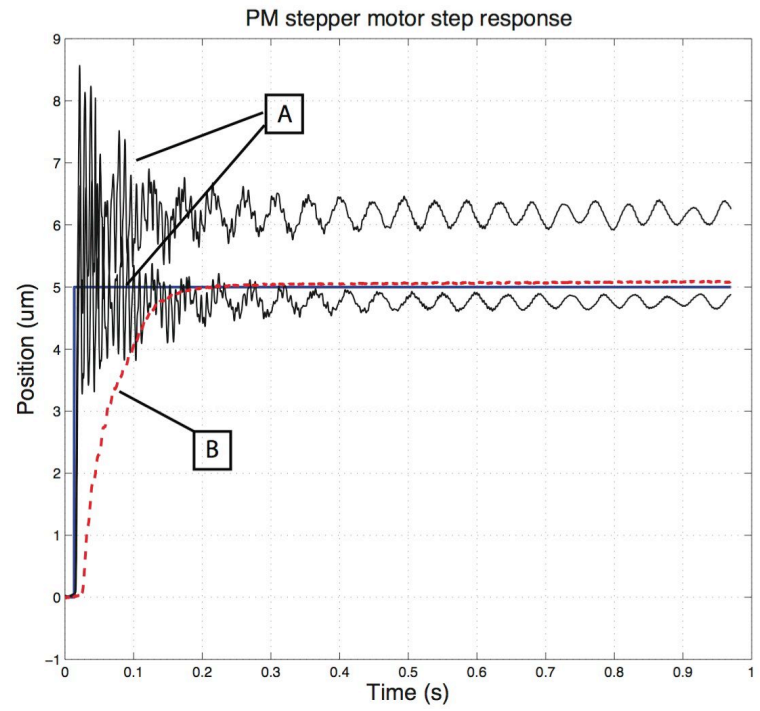

Fig. 6. Coarse actuator: comparison of the two different control approaches. A) Two measured responses to a $5 \mu \mathrm{m}$ step of the approach using the microstepping electronic driver: inaccurate, because of uncontrolled nonlinearities and oscillations, with no control on the trajectories and large incertitude in the result.

B) A realization of the approach using direct coil-voltage control to the same $5 \mu \mathrm{m}$ step: requires a more elaborate control algorithm, but with controlled and predictable trajectories.

The resonant frequency is well damped by the PZT dedicated electronics and, therefore, a classical proportional integral (PI) controller is well suited for controlling this fine stage. Figure 7(a) represents the Bode magnitude analysis of this transfer function, which was used to tune the parameters of our controller.

So as to improve the tracking performances of the complete system, a feed-forward prediction of the stepper movement is added to the piezoelectric actuator control voltage. This prediction is based on the PM stepper-motor control variable $x$ (see Equation 9) and on the reference signal fed to the stepper controller. The predicted movement of the coarse stage is scaled with the inverse static gain of the identified model of the piezoelectric actuator and added in feed forward to its control signal (see Figure 7(b)) to compensate for the real movement of the coarse stage. This guarantees better stepper movement rejection.

Moreover, the PM stepper-motor controller based on direct coil-voltage control presented in Section (III-B) is particularly well suited for this kind of prediction, as the instantaneous position of the stepper is well known through the control variable $x$. The feed-forward structure proposed here reduces drastically the overall position tracking error.

\section{Proposed GLOBAL CONTROL STRATEGY}

The main property of a double-stage system is its possibility to be considered as two systems acting in parallel and simultaneously on a single output. Moreover, a structural property of such double-stage systems is the possibility to represent the 


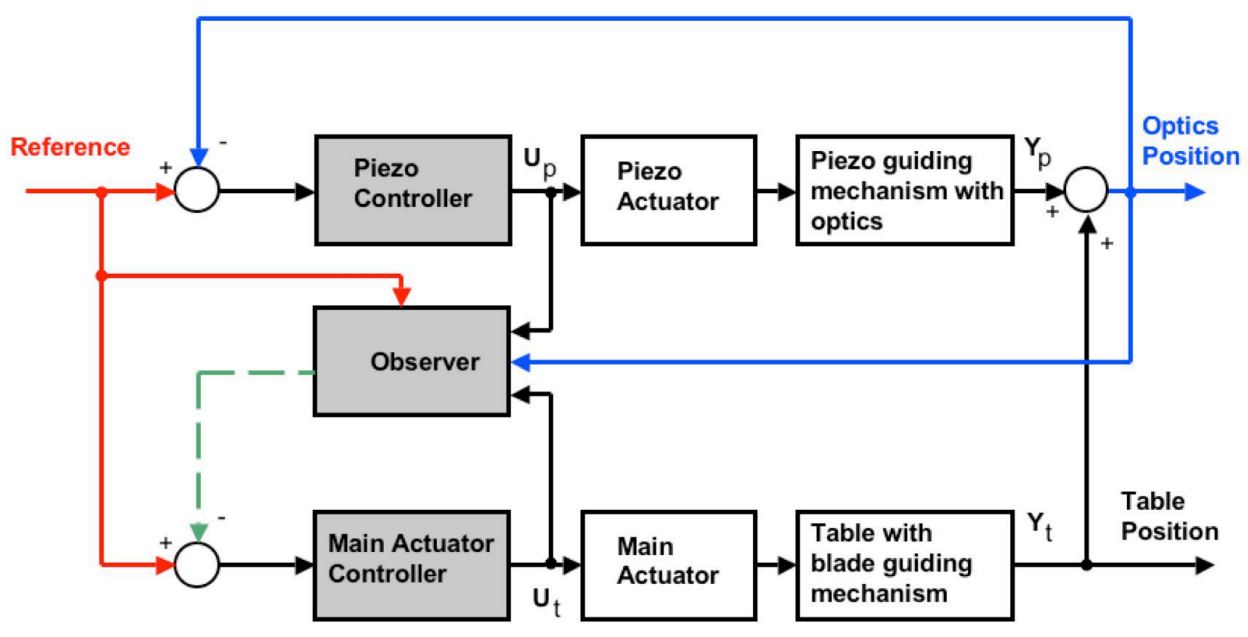

Fig. 8. General control structure of the double-stage system with a single metrology. 1) Combination of the two actuators on the controlled output (optics position). 2) A single metrology sensor measures the overall position. 3) A partial observer gives the feedback information for the coarse stage.

interactions of the actuators on the output by a combination of their independent effects on the controlled translation axis, as illustrated in Figure 8. In this figure, the overall output is composed of a linear combination of the two-stage independent outputs.

So as to avoid the use of supplementary sensors to determine their relative effects on the measured output, a partial observer is proposed to close the coarse loop. Closing both loops directly with the overall measured position does indeed bring the short-stroke piezoelectric actuator to an unacceptable (the bandwidth is not covered anymore) saturation, before the slower coarse one really moves. The goal of this combination is to drive simultaneously both actuators to overcome their drawbacks. A partial observer was introduced to close the coarse loop and maintain the fast piezoelectric in its midposition as much as possible.

This parallel control approach, illustrated in Figure 8, decoupling the two stages with an observer has also been introduced for Hard Disk Drive applications (see [17], [19], [22] for examples).

Furthermore, it must be underlined that the fine stage loop is intentionally closed on the combined effect of both stages, in order to ensure an efficient tracking of the output optics position. Neither a model nor an observer is placed on its feedback (see upper blue loop in Figure 8), so as to ensure the overall tracking of the real position and thereby avoiding any model mismatch error (see Figure 8).

The partial observer previously mentioned estimates the position of the coarse stage driven by the PM stepper motor based on the piezoelectric fine actuator model $P$, its voltage input $U_{p}$, the reference signal $r$ and the overall measured position $y$ (see Figure 9).

The model $P$ of the piezoelectric stack actuator can be obtained using classical identification procedures. However, in case there is an intrinsic bandwidth difference between the actuators (one order of magnitude for example) or when this difference is imposed by the controllers, a simple static approximation of the fast actuator is sufficient (see Figure 10). The error induced by the simplification in the model is appearing only at high frequencies, larger than the bandwidth of the coarse system. The static partial observer estimates correctly the position at low frequencies. Thus, the neglected dynamical impact on the tracking is really secondary, since the fine stage piezoelectric (whose bandwidth is much larger) rejects easily any possible resulting error of the coarse stage.

Once this coarse-table position is estimated, the coarse loop can be closed (see Figure 8) on its estimated position. Thus, even if the piezoelectric actuator cancels the overall error, the coarse table driven by the PM stepper motor cancels its own relative error. Therefore, the stepper action brings back slowly the piezoelectric to its mid-position, while avoiding piezoelectric saturation.

\section{EXPERIMENTAL RESULTS}

A prototype has been tested through a realization of a stochastic Kolmogorov signal, with a frequency content of up to $200 \mathrm{~Hz}$, as a reference. This signal is representative of the atmospheric perturbations encountered, the rejection of which should be assured by the currently developed differential delay line.

As a main result, the proposed global control structure based on a single metrology to close both loops combines optimally both stages. The global control structure presented in Section V (including the coarse stage feedback based on the partial observer) has been validated with the two different PM stepper motor control approaches. No special unwanted oscillations between the two stages and no time-drift behavior due to the partial observer have been observed: this validates our control structure. Moreover, thanks to the proposed double stage control loop structure, the piezoelectric stack actuator 
never reaches its saturation limits while the complete system is working (see Figure 11).

The neglected dynamics of the piezoelectric appearing in the partial observer does not have any impact on the whole behavior, nor on the tracking accuracy of the complete system. This result holds as long as the fine stage controller feedback compares directly the reference to the overall measured output rejecting all uncertainties and coarse stage model errors.

The two different permanent-magnet stepper-motor control approaches and their impact on the tracking accuracy of the complete double-stage system are compared below. On the one hand, the classical electronic driver is used to drive the coils of the motor. The coarse loop is closed through this driver and the dead-zone-like nonlinear controller for the coarse stage maintains the fine stage in its mid-position. On the other hand, the integral controller which controls directly the voltage of the coils of the same PM stepper motor is designed and compared to the first one.

The two different implemented PM stepper motor controllers are compared through the remaining tracking error

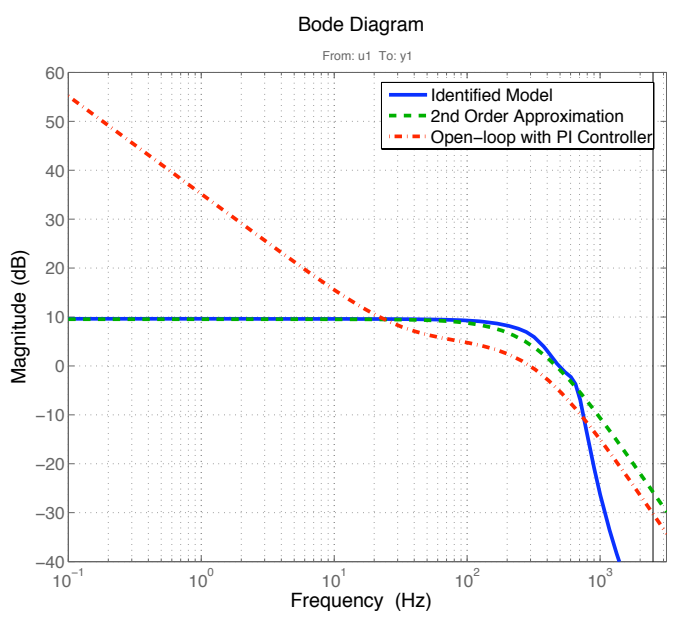

(a) Identified model of the piezoelectric stack actuator S-325, with its second-order approximation and the tuned open-loop behavior with the PI controller. The estimated bandwidth is approximately $300 \mathrm{~Hz}$ and the static gain is of approximately 3 (corresponding to a $10 \mathrm{~V}$ input to $30 \mu \mathrm{m}$ output scaling).

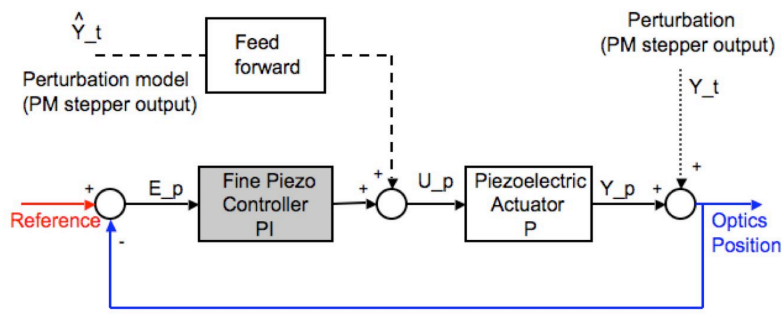

(b) Control strategy of the fine piezoelectric actuator stage. A classical PI controller with a feed-forward prediction of the perturbation introduced by the stepper is used.

Fig. 7. Fine stage piezoelectric stack actuator model and control law.

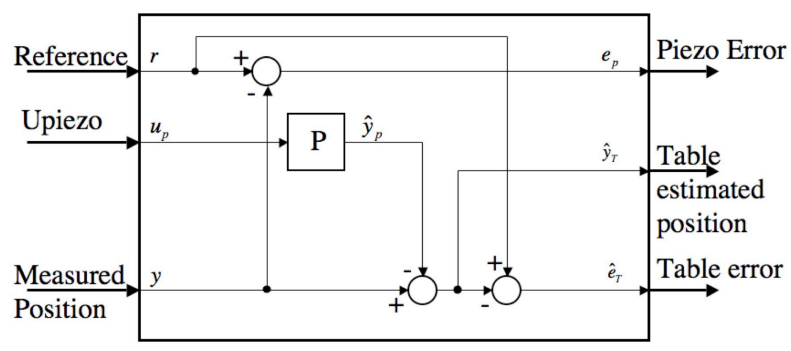

Fig. 9. Partial observer as relative position estimator based on the piezoelectric actuator's model. $P$ represents the fine-actuator model.

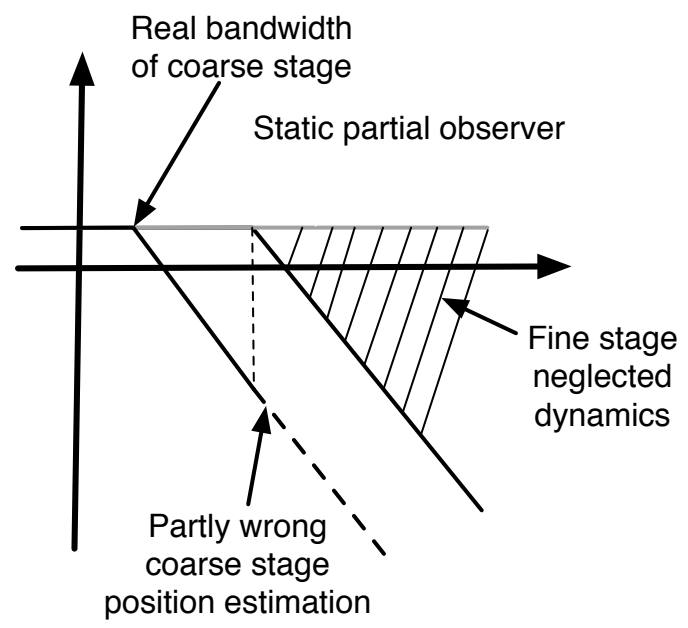

Fig. 10. Partial static observer. The piezoelectric neglected dynamics influences the position estimation of the coarse stage only at high frequencies (larger than its own bandwidth).

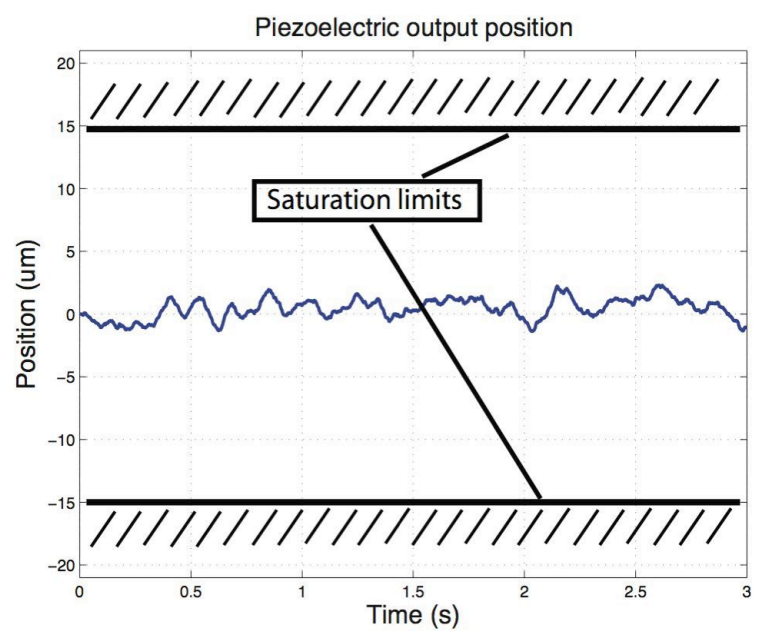

Fig. 11. The piezoelectric actuator controlled output never reaches its saturation limits during trajectory tracking.

(see Table I. Furthermore, the position tracking results of the two different approaches are illustrated in Figure 12 for the classical electronic driver with a nonlinear dead-zone-like 
TABLE I

TRACKING ERROR WITH A KOLMOGOROV SIGNAL AS EXCITATION

\begin{tabular}{|c|c|c|}
\hline Controller & Tracking Error rms & $\begin{array}{c}\text { Peak Tracking } \\
\text { Error } \mathrm{nm}\end{array}$ \\
\hline \hline $\begin{array}{c}\text { Classical electronic driver } \\
\text { with dead-zone controller }\end{array}$ & $e<30 \mathrm{~nm} \mathrm{rms}$ & $|e|<200 \mathrm{~nm}$ \\
\hline $\begin{array}{c}\text { Direct coil control } \\
\text { with integral controller }\end{array}$ & $e<8 \mathrm{~nm} \mathrm{rms}$ & $|e|<30 \mathrm{~nm}$ \\
\hline
\end{tabular}

controller approach and, in Figure 13, for the direct coilvoltage control with an integral controller.

Relative large error spikes $(200 \mathrm{~nm})$ are periodically visible for the first approach. These spikes (see the (*) in Figure 12) correspond to the activation of the PM stepper motor. The movement of the coarse stage is almost entirely seen as an unmodeled perturbation for the fine stage controller (as stated in Section III, this movement is hardly predictable). Therefore, this unpredicted perturbation results in large instantaneous errors as the fine stage controller needs some time to reject it efficiently.

The second approach, which relies on direct coil-voltage control, results undoubtedly in better performance: the rms residual tracking error is as low as the measurement noise ( $\mathrm{E}<8 \mathrm{~nm} \mathrm{rms}$ ) and the movement of the coarse stage does not result in any visible tracking error of the overall system. These very impressive results (i.e. just $8 \mathrm{~nm}$ rms tracking error up to $300 \mathrm{~Hz}$ ) are obtained with just a small increase in the algorithm complexity, because the proposed algorithm implements a classical integral controller. The feed-forward structure based on the estimated instantaneous position of the stepper is the leading element that gives these outstanding results in stochastic trajectory tracking, obtained with the second control method of the PM stepper motor. In fact, as the coarse stage position gets close to the control variable $x$ (to the rotation to linear conversion ratio $\gamma$ factor), the prediction of the dynamical movements followed by the coarse stage are well estimated and easily canceled by the fine stage.

\section{CONCLUSIONS}

This paper presents a global control structure applied to a double-stage mechanical system conceived in order to reach nanometer-accuracy dynamic positioning over a large stroke range. The key control difficulty was the strong stroke limitation of the fine actuator which needed the addition of a coarse positioning stage.

The proposed control strategy controls both actuators simultaneously, contrary to a hybrid control strategy. This ensures highly accurate tracking of the reference by the whole system, which was demonstrated on a real testbed and shown to be extremely efficient. In order to render the mutual actions of the actuators effective in the positioning of the final position reference, a suitable observer-based controller was designed. The partial observer together with the coarse control loop tends to maintain the fine actuator close to its median position as much as possible, which is difficult to ensure when using a purely hybrid strategy.

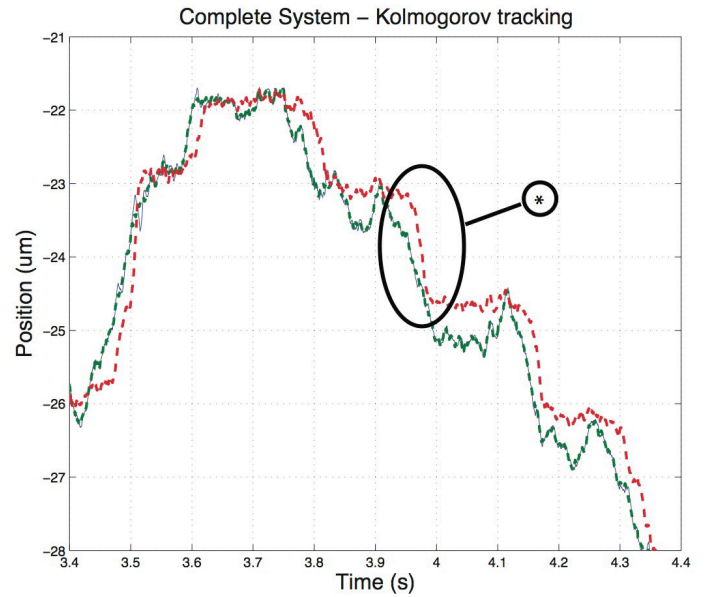

(a) Tracking of a Kolmogorov realization: classical electronic driver approach. The continuous line is the position reference and well superimposed is the measured final output; the dash-dotted line is the coarse stage estimated position.

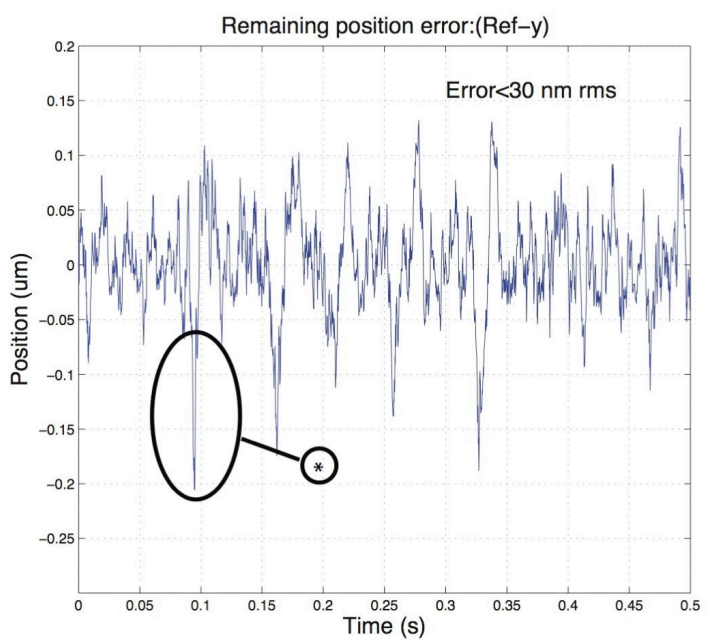

(b) Remaining tracking error.

Fig. 12. System response to a realization of the stochastic Kolmogorov process with a frequency content of up to $200 \mathrm{~Hz}$. Results obtained with the classical electronic PM stepper motor driver and a dead-zone-like controller, giving a residual rms error less than $30 \mathrm{~nm}$ rms, but with spikes up to 200 nm whenever the stepper moves (see $(*)$ on the Figure for example).

Two different solutions implementing two different stepper control approaches were tested with success on the doublestage mechanical system, using the same global control structure. On the one hand, a classical electronic driver was used to control the PM stepper motor through a nonlinear deadzone-like controller. On the other hand, a direct control of the coil voltage was used to control the PM stepper motor through a simple integrator controller. Both approaches were compared using experimental data. As was clearly demonstrated (although at the cost of a slight increase in the control algorithm complexity), the performance of the second method was far better. Therefore, this last method should be preferred. 


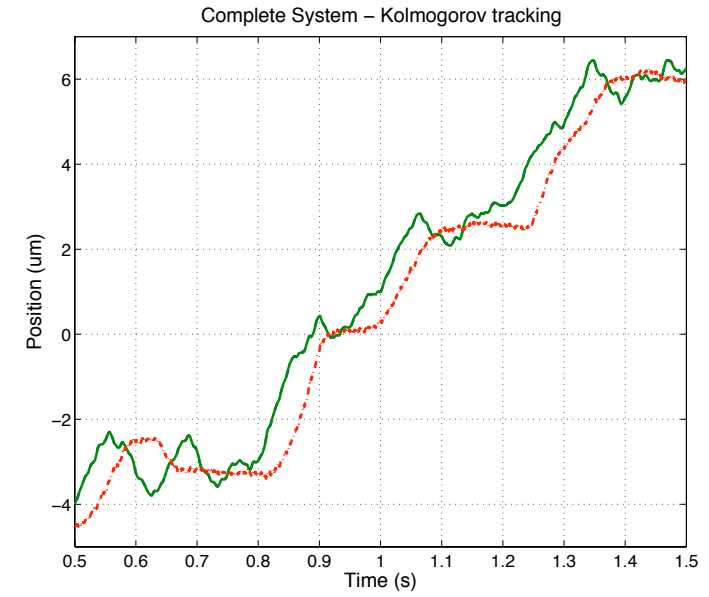

(a) Tracking of a Kolmogorov realization: direct voltage control. The continuous line is the position reference and perfectly superimposed is the measured final output; the dash-dotted line is the coarse stage estimated position.

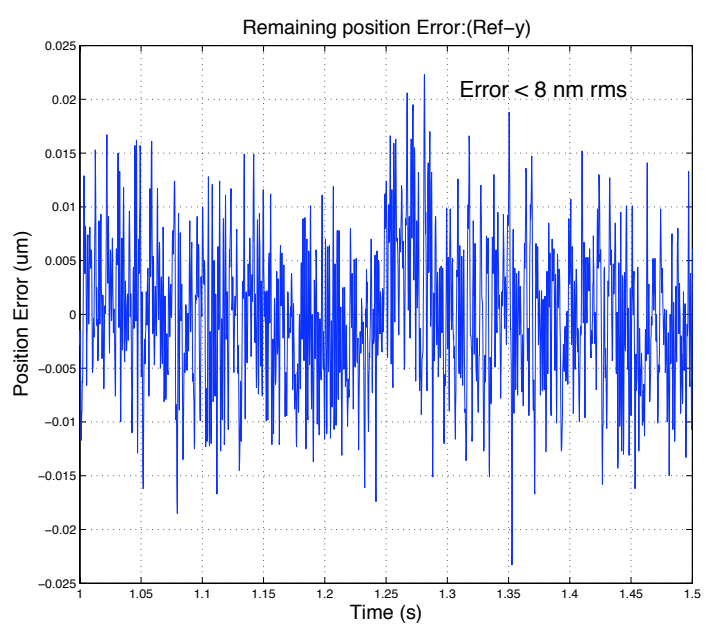

(b) Remaining tracking error.

Fig. 13. System response to the same realization of the stochastic Kolmogorov process with a frequency content of up to $200 \mathrm{~Hz}$. Results obtained with the direct coil-voltage control of the PM stepper motor and the integral controller, giving a residual $\mathrm{rms}$ error less than $8 \mathrm{~nm} \mathrm{rms}$ and a maximum instantaneous error of $30 \mathrm{~nm}$.

As a final conclusion, the presented double-stage control structure based on a single metrology feedback has been successfully implemented to reach the required accuracy and bandwidth.

\section{ACKNOWLEDGMENTS}

The authors would like to thank the ESO (European Southern Observatory) and the consortium working on the Differential Delay Line for the VLTI at ESO, including the Geneva Observatory, the University of Leiden, the MPIA (Max-PlanckInstitut for Astronomy), the Institute of Microtechnics of Neuchâtel and the EPFL (École Polytechnique Fédérale de Lausanne).

\section{REFERENCES}

[1] Chih-Lyang Hwang, Yung-Ming Che, J. K. O. Sin and Chau Jan, "Trajectory tracking of large-displacement piezoelectric actuators using a nonlinear observer based variable structure control," in IEEE Trans. on Control Systems Technology, vol.13, pp. 56-66, Jan., 2005.

[2] Brian A. Awabdy, Wu-Chu Shih and M. Auslander, "Nanometer Positioning of a Linear Motion Stage Under Static Load, " in IEEE Trans. on Mechatronics, vol. 3, pp. 113-119, June, 1998.

[3] Yung-Tien Liu, Rong-Fong Fung and Tai-Kun Huang, "Dynamic response of a precision positioning table impacted by a soft-mounted piezoelectric actuator, " in Precision Engineering, vol. 28, pp. 252-260, 2004.

[4] Martin Dimmler, Ulf Holmberg and Roland Longchamp, "Hysteresis Compensation of Piezo Actuators," in Proceedings of European Control Conference, Karlsruhe, Germany, 1999.

[5] Hongzhong Liu, Bingheng Lu, Yucheng Ding, Yiping Tang and Dichen $\mathrm{Li}$, "A motor-piezo actuator for nano-scale positioning based on dual servo loop and nonlinearity compensation" in Inst. of Physics Publishing, Journal of Micromechanics and Microenginering, vol. 13, 2003.

[6] Bi Zhang and Zhenqi Zhu, "Developing a Linear Piezomotor With Nanometer Resolution and High Stiffness," in IEEE/ASME Trans. on Mechatronics, vol. 2, 1997.

[7] S. Devasia, "Nonlinear Inversion-Based Output tracking," in IEEE Trans. on Automatic Control, Vol. 41, 1996.

[8] S. Salapaka, A. Sebastien, J. P. Cleveland and M. V. Salapaka, "High bandwidth nano-positioner: A robust control approach," in Rev. Sci. Instrum., vol. 73, 2002.

[9] Takashi Kenjo and Akira Sugawara, "Stepping Motors and their Microprocessor Controls," Oxford Science Publications, Second Edition, 1994.

[10] L. Chrifi-Alaoui and A. Lebrun, " $H_{\infty}$ Feedback Control of a Permanent Magnet Stepper Motor", in Oxford Science Publications, Second Edition, 1994.

[11] Marc Bodson, John N. Chiasson, Robert T. Novotnak and Ronald B. Rekowski, "High-Performance Nonlinear Feedback Control of a Permanent Magnet Stepper Motor," in IEEE Trans. on Control Systems Technology, vol. 1, No. 1, March 1993.

[12] Marc Bodson and John Chiasson, "Application of Nonlinear Control Method to the Positioning of a Permanent Magnet Stepper Motor," in Proceedings of the Conference on Decision and Control, 1989.

[13] Michael Aiello, Ronald Rekowski, Marc Bodson John Chiasson and David Schuerer, "Experimental results of using an exact linearization controller on a PM Stepper Motor," in Proceedings of the Conference on Decision and Control, 1990.

[14] M. Zribi and J. Chiasson, "Position Control of a PM Stepper Motor by Exact Linearization," in IEEE Trans. on Automatic Control, vol. 36, No. 5, 1991.

[15] Pragasen Pillay and Ramu Krishnan, "Modeling, Simulation, and Analysis of Permanent-Magnet Motor Drives, Part I: The Permanet-Magnet Synchronous Motor Drive," in IEEE Trans. on Industry Applications, vol. 25, No. 2, 1989.

[16] Herbert Sira-Ramirez, "Trajectory planning in the regulation of a PM stepper motor: A combined passivity and flatness approach," in Proceedings of the American Control Conference, 2000.

[17] Abdullah Al Mamun, Iven Mareels, T. H. Lee and Arthur Tay, "Dual Stage Actuator Control in Hard Disk Drive-A Review," in $29^{\text {th }}$ Annual Conference of the IEEE, vol. 3, 2003.

[18] Steven J. Schroeck, William C. Messner and Robert J. McNab, "On Compensator Design for Linear Time-Invariant Dual-Input SingleOutput Systems," in IEEE/ASME Trans. on Mechatronics, vol. 6, 2001.

[19] Masahito Kobayashi and Roberto Horowitz, "Track Seek Control for Hard Disk Dual-Stage Servo Systems," in IEEE Trans. on Magnetics, vol. $37,2001$.

[20] Xiaoping Hu, Wei Guo, Tony Huang and Ben M. Chen, "DiscreteTime LQG/LTR Dual-Stage Controller Design and Implementation for High Track Density HDDs," in Proceedings of the American Control Conference, 1999.

[21] M. Rotunno and R. A. de Callafon, "Fixed Order $H_{\infty}$ Control Design for Dual-Stage Hard Disk Drives," in Proceedings of the $39^{\text {th }}$ IEEE Conference on Decision and Control, 2000.

[22] S. Koganezawa, Y. Uematsu and T. Yamada, "Dual-Stage Actuator System for Magnetic Disk Drives Using a Shear Mode Piezoelectric Microactuator," in IEEE Trans. on Magnetics, vol. 35, No. 2, 1999. 